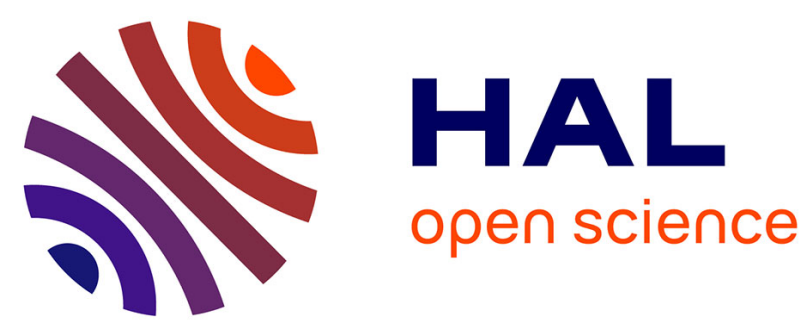

\title{
Reciprocity, unitarity, and time-reversal symmetry of the S matrix of fields containing evanescent components
}

\author{
R. Carminati, J J Sáenz, J.-J Greffet, M. Nieto-Vesperinas
}

\section{To cite this version:}

R. Carminati, J J Sáenz, J.-J Greffet, M. Nieto-Vesperinas. Reciprocity, unitarity, and timereversal symmetry of the $\mathrm{S}$ matrix of fields containing evanescent components. Physical Review A : Atomic, molecular, and optical physics [1990-2015], 2000, 62, pp.012712-012718 10.1103/PhysRevA.62.012712 . hal-01624615

\section{HAL Id: hal-01624615 https://hal.science/hal-01624615}

Submitted on 27 Oct 2017

HAL is a multi-disciplinary open access archive for the deposit and dissemination of scientific research documents, whether they are published or not. The documents may come from teaching and research institutions in France or abroad, or from public or private research centers.
L'archive ouverte pluridisciplinaire HAL, est destinée au dépôt et à la diffusion de documents scientifiques de niveau recherche, publiés ou non, émanant des établissements d'enseignement et de recherche français ou étrangers, des laboratoires publics ou privés. 


\title{
Reciprocity, unitarity, and time-reversal symmetry of the $S$ matrix of fields containing evanescent components
}

\author{
R. Carminati, ${ }^{1}$ J. J. Sáenz, ${ }^{2}$ J.-J. Greffet, ${ }^{1}$ and M. Nieto-Vesperinas ${ }^{3}$ \\ ${ }^{1}$ Laboratoire d'Energétique Moléculaire et Macroscopique, Combustion, Ecole Centrale Paris, \\ Centre National de la Recherche Scientifique, 92295 Châtenay-Malabry Cedex, France \\ ${ }^{2}$ Departamento de Física de la Materia Condensada and Instituto de Ciencia de Materiales "Nicolás Cabrera," \\ Universidad Autónoma de Madrid, Cantoblanco, 28049 Madrid, Spain \\ ${ }^{3}$ Instituto de Ciencia de Materiales de Madrid, Consejo Superior de Investigaciones Científicas, \\ Cantoblanco, 28049 Madrid, Spain
}

(Received 7 February 2000; published 16 June 2000)

\begin{abstract}
We derive general relationships of the $S$ matrix of fields containing evanescent components. Our formalism covers time-independent quantum scattering as well as scattering of classical scalar waves. We show that reciprocity, energy (or probability) conservation, and time-reversal symmetry in the presence of evanescent waves lead to relationships that extend the well-known relations previously derived in asymptotic scattering. On this basis, we discuss the link between reciprocity and time-reversal symmetry. We also address the experimental feasibility of time reversal of a field containing evanescent components.
\end{abstract}

PACS number(s): 03.65.Nk, 03.50.-z, 42.25.Fx, 11.55.-m

\section{INTRODUCTION}

The scattering matrix ( $S$ matrix) was introduced by Heisenberg to describe a scattering process without any assumption about the details of the interaction [1]. In this formalism, the process is thought of as a transformation of an incoming state $\Psi_{\text {in }}$ into an outgoing state $\Psi_{\text {out }}$, which describe the system far away from the interaction potential. Hence, the $S$ matrix describes the scattering process asymptotically. The mathematical transcription of this transformation is an operator relationship $\Psi_{\text {out }}=\mathbf{S} \Psi_{\text {in }}$, where $\mathbf{S}$ is called the $S$ matrix $[2,3]$. It is well known that the $S$ matrix exhibits some properties that are independent of the specific problem under study. In particular, it is unitary and symmetric, these two properties reflecting probability (or energy) conservation in elastic scattering and reciprocity, respectively [3]. The general aim being to get maximum information about the $S$ matrix with minimum knowledge about the interaction itself, other properties may be derived, based, for example, on dispersion relations and causality conditions [4]. The existence of such general properties of the $S$ matrix is the reason why it has become a fundamental tool in most areas of theoretical physics, e.g., in quantum scattering [2,3], in particle physics [5], in field theory, and in statistical physics [6]. Its definition and its use have also been extended to scattering of classical (acoustic and electromagnetic) waves $[7,8]$. For example, the $S$ matrix has become a fundamental tool (as well as a practical one) to compute scattered fields in physical optics [9]. This formalism has also found a wide range of applications with the development of randommatrix theory [10], which has recently acquired renewed interest through its use in quantum- and classical-wave transport in random media $[11,12]$.

The $S$ matrix was originally defined as an operator acting on asymptotic states. In scattering by a time-independent potential (we shall restrict our discussion to this case), this means that the $S$ matrix relates the far-field amplitudes of the incoming and outgoing fields $[2,7,8]$. Nevertheless, in the last ten years, new effects have been observed and new tech- niques developed that involve scattering and/or direct measurement of evanescent waves (near fields). For example, evanescent-wave scattering is involved in the emission process of an atom or a molecule close to a surface [13] or in the surface-plasmon-polariton (SPP) mechanism which leads to the enhanced backscattering of light on slightly rough metal surfaces [14]. It is also the basic principle of near-field scanning probe microscopies, using either electron [15] or photon [16] tunneling. Modeling the image formation in scanning near-field optical micoscopy (SNOM) requires a precise description of a mechanism involving scattering of evanescent waves [17]. The advent of SNOM has also allowed a direct experimental study of SPP excitation and scattering [18] and Anderson localization of surface excitations [19] and stimulated theoretical works on SPP scattering by surface roughness or localized objects [20]. In all these fields, the description of the coupling between an incident evanescent wave and a scattered propagating or evanescent wave is of fundamental interest. An $S$-matrix formalism, with a definition including the near-field components, should be very useful in this context. The $S$ matrix also provides a useful formalism to discuss time reversal of wave fields, and especially its link with reciprocity (symmetry of the $S$ matrix) and probability or energy conservation (unitarity). In particular, the question of time reversal of fields containing evanescent components has recently received increasing attention, with the demonstration of phase conjugation of optical near fields [21] and of time reversal of acoustic waves [22]. In this last case, the $S$ - matrix formalism was used to discuss the properties of a time-reversal acoustic cavity, without taking into account the role of evanescent waves [23]. Nevertheless, the question of subwavelength focusing of a field by time reversal was raised. This is an important issue, whose discussion requires the use of a formalism including the evanescent components of the field.

Finding general properties of the $S$ matrix, extended to evanescent waves, is of major importance in understanding and modeling all phenomena and devices involving nearfield scattering. To our knowledge, this problem has received 


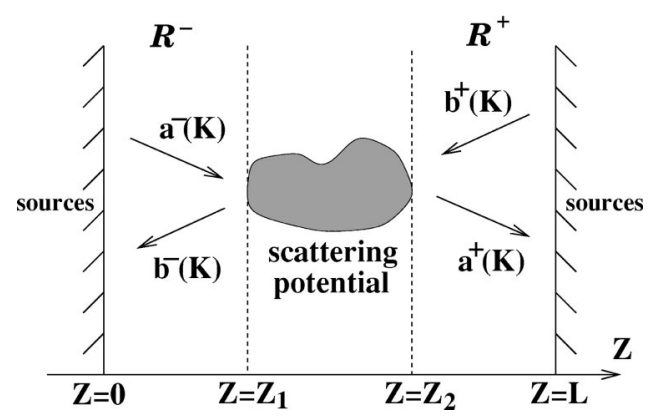

FIG. 1. Scattering geometry and notation.

little attention until now, except in electromagnetic waveguide theory where reciprocity and unitarity relations for the $S$ matrix in the presence of evanescent modes have been briefly discussed [24]. In this work, we concentrate on reciprocity, unitarity, and time-reversal symmetry in the framework of scattering from a localized potential, in the presence of evanescent waves in both the incoming and outgoing fields. The formalism we use covers time-independent quantum scattering as well as scattering of classical scalar waves. In Sec. II, we define the $S$ matrix based on the angular representation of the field, sometimes called the partitioned $S$ matrix in the literature $[9,25]$. In Sec. III, we give a general derivation of the $S$ matrix reciprocity for scalar (quantum and classical) fields, in the presence of evanescent waves. In Sec. IV, we show that energy (or probability) conservation in scattering of fields containing evanescent components leads to generalized unitarity relations of the $S$ matrix. These relations extend those previously derived for source-free fields (i.e., fields without evanescent components) $[9,25]$, and those obtained in electromagnetic waveguide theory [24]. In Sec. $\mathrm{V}$, we address the problem of time-reversal symmetry of wave fields containing evanescent components. We show that the time-reversal invariance condition leads to a different relationship for the $S$ matrix. On this basis, the link between reciprocity, unitarity, and time-reversal invariance is discussed. This problem is of fundamental and practical importance, due, for example, to its potential application to time reversal of acoustic waves [22]. Finally, we give a summary and a general conclusion in Sec. VI.

\section{DEFINITION OF THE $S$ MATRIX}

Let us consider the scattering problem depicted in Fig. 1. The regions $0<z<z_{1}$ and $z_{2}<z<L$, denoted by $\mathcal{R}^{-}$and $\mathcal{R}^{+}$, respectively, are assumed to be of constant potential, so that the wave field in these regions obeys the timeindependent wave equation

$$
\nabla^{2} \Psi(\mathbf{r})+k^{2} \Psi(\mathbf{r})=0,
$$

where $\mathbf{r}=(x, y, z)$. In Eq. (1), $\Psi(\mathbf{r})$ is either the timeindependent wave function of a state of energy $E\left[k^{2}\right.$ $=2 m / \hbar^{2}(E-V)$, where $V$ is the potential and $m$ the mass of the particle], or a monochromatic classical wave of frequency $\omega(k=\omega / c$, where $c$ is the phase velocity in the medium). The wave number $k^{2}$ is real, but can be negative, e.g., in a tunneling barrier. The scattering potential is as- sumed confined within the strip $z_{1}<z<z_{2}$, and independent of the field (linear scattering). The regions $z<0$ and $z>L$ contain sources, the presence of which, at finite distance from the scattering region, is necessary to ensure the existence of incident evanescent waves in both $\mathcal{R}^{-}$and $\mathcal{R}^{+}$. The evanescent waves explicitly appear when the angularspectrum representation (or plane-wave expansion) of the field is used. In this representation, the fields $\Psi^{-}$in $\mathcal{R}^{-}$and $\Psi^{+}$in $\mathcal{R}^{+}$are written [9]

$$
\begin{aligned}
\Psi^{ \pm}(\mathbf{r})= & \int a^{ \pm}(\mathbf{K}) \exp (i \mathbf{K} \cdot \mathbf{R}+i \gamma z) d^{2} \mathbf{K} \\
& +\int b^{ \pm}(\mathbf{K}) \exp (i \mathbf{K} \cdot \mathbf{R}-i \gamma z) d^{2} \mathbf{K},
\end{aligned}
$$

where $\gamma(\mathbf{K})=\sqrt{k^{2}-K^{2}}$ for $K^{2} \leqslant k^{2}$ (homogeneous or propagating components) and $\gamma(\mathbf{K})=i \sqrt{K^{2}-k^{2}}$ for $K^{2}>k^{2}$ (inhomogeneous or evanescent components). We use the notations $\mathbf{R}=(x, y)$ and $K=|\mathbf{K}|$. Except when the integration domain is specified, all integrals in this paper are extended to $-\infty$ $<\left(K_{x}, K_{y}\right)<+\infty$. Note that Eq. (2) is a representation of the field valid in regions $\mathcal{R}^{-}$and $\mathcal{R}^{+}$where $|z|$ remains finite, so that there is no divergence of evanescent waves when $|z|$ increases.

In the angular-spectrum representation (2), the partitioned $S$ matrix relates the outgoing vector $\Psi^{\text {out }}(\mathbf{K})$ $=\left[b^{-}(\mathbf{K}) a^{+}(\mathbf{K})\right]$ to the incoming vector $\Psi^{\text {in }}(\mathbf{K})$

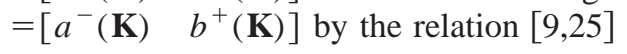

$$
\Psi^{\text {out }}(\mathbf{K})=\int \mathbf{S}\left(\mathbf{K}, \mathbf{K}^{\prime}\right) \Psi^{\text {in }}\left(\mathbf{K}^{\prime}\right) d^{2} \mathbf{K}^{\prime}
$$

where $\mathbf{S}$ is a $2 \times 2$ matrix, sometimes called the partitioned $S$ matrix $[9,25]$, which can be written in the form

$$
\mathbf{S}\left(\mathbf{K}, \mathbf{K}^{\prime}\right)=\left[\begin{array}{ll}
r\left(\mathbf{K}, \mathbf{K}^{\prime}\right) & \tau\left(\mathbf{K}, \mathbf{K}^{\prime}\right) \\
t\left(\mathbf{K}, \mathbf{K}^{\prime}\right) & \rho\left(\mathbf{K}, \mathbf{K}^{\prime}\right)
\end{array}\right] .
$$

The four elements $r, t, \rho, \tau$ have the meaning of generalized reflection and transmission coefficients $[9,25]$. Their definition can easily be extended to vector fields, as in electromagnetic scattering. In this case, the four coefficients become tensor operators [26].

\section{RECIPROCITY RELATION}

For incoming and outgoing fields without evanescent components (source-free fields), reciprocity and unitarity relations for the partitioned $S$ matrix are well established $[9,25]$. They were derived as a consequence of the symmetry and unitarity of the asymptotic (far-field) $S$ matrix. Extending these relations to general wave fields with evanescent components requires a different procedure. Note that reciprocity of evanescent waves was derived previously in specific cases, such as electromagnetic waveguide theory [24], electromagnetic (vector field) scattering [26], and elasticwave scattering at a solid-solid interface [27]. In these examples, a suitable formulation of the reciprocity theorem was used for each particular case. In this paper, we give a proof of reciprocity of the $S$ matrix for scattering of both homoge- 
neous and evanescent scalar waves from a localized potential, starting from a general formulation of reciprocity valid for any kind of scalar wave.

Let $\Psi_{1}$ and $\Psi_{2}$ be two fields that are solutions of the scattering problem depicted in Fig. 1. With reference to Fig. 2 , let us consider the volume $V$ delimited by the closed surface $\Sigma$ composed of two planes $z=z^{-}$and $z=z^{+}$and a sphere of radius $R$ centered at the center of the potential region. The application of Green's second identity leads to

$$
\int_{V}\left(\Psi_{2} \Delta \Psi_{1}-\Psi_{1} \Delta \Psi_{2}\right) d V=\int_{\Sigma}\left(\Psi_{2} \frac{\partial \Psi_{1}}{\partial n}-\Psi_{1} \frac{\partial \Psi_{2}}{\partial n}\right) d S
$$

where $\partial / \partial n=\mathbf{n} \cdot \nabla$ and $\mathbf{n}$ is the outward normal on the surface $\Sigma$. Both $\Psi_{1}$ and $\Psi_{2}$ satisfy Eq. (1) in $\mathcal{R}^{-}$and $\mathcal{R}^{+}$, so that the integrand in the left-hand side in Eq. (5) vanishes. Moreover, in the far-field asymptotic limit $|k| r \rightarrow \infty$, one has $\partial \Psi_{j} / \partial r=i k r^{-1} \Psi_{j}$, so that $\Psi_{2} \partial \Psi_{1} / \partial n-\Psi_{1} \partial \Psi_{2} / \partial n$ vanishes identically on the sphere surface when its radius $R$ tends to infinity. Finally, Eq. (5) leads to the following equality:

$$
\begin{aligned}
\int_{z=z^{-}} & \left(\Psi_{2} \frac{\partial \Psi_{1}}{\partial z}-\Psi_{1} \frac{\partial \Psi_{2}}{\partial z}\right) d^{2} \mathbf{R} \\
& =\int_{z=z^{+}}\left(\Psi_{2} \frac{\partial \Psi_{1}}{\partial z}-\Psi_{1} \frac{\partial \Psi_{2}}{\partial z}\right) d^{2} \mathbf{R} .
\end{aligned}
$$

Equation (6) is a scalar version of Lorentz's reciprocity theorem, originally derived for the electromagnetic field [28]. In order to obtain a reciprocity theorem for the $S$ matrix, we introduce the angular-spectrum representation Eq. (2) of the fields $\Psi_{1}$ and $\Psi_{2}$ into Eq. (6). After some algebra, one obtains the following expression:

$$
\begin{aligned}
& \iint\left[a_{1}^{-}(\mathbf{K}) \quad b_{1}^{+}(\mathbf{K})\right]\left\{\gamma(\mathbf{K}) \mathbf{S}\left(\mathbf{K}, \mathbf{K}^{\prime}\right)\right. \\
& \left.-\gamma\left(\mathbf{K}^{\prime}\right) \mathbf{S}^{T}\left(-\mathbf{K}^{\prime},-\mathbf{K}\right)\right\}\left[\begin{array}{l}
a_{2}^{-}\left(\mathbf{K}^{\prime}\right) \\
b_{2}^{+}\left(\mathbf{K}^{\prime}\right)
\end{array}\right] d^{2} \mathbf{K} d^{2} \mathbf{K}^{\prime}=0,
\end{aligned}
$$

where the superscript $T$ denotes the transposed matrix. Because Eq. (7) must be satisfied for any incoming vectors

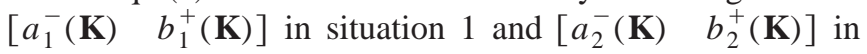
situation 2 , one finally obtains

$$
\gamma(\mathbf{K}) \mathbf{S}\left(\mathbf{K}, \mathbf{K}^{\prime}\right)=\gamma\left(\mathbf{K}^{\prime}\right) \mathbf{S}^{T}\left(-\mathbf{K}^{\prime},-\mathbf{K}\right) .
$$

Equation (8) is valid for $0<|\mathbf{K}|<+\infty$ and $0<\left|\mathbf{K}^{\prime}\right|<+\infty$, i.e., for propagating and evanescent waves. Note that the presence of the factors $\gamma$ in Eq. (8) is a consequence of the definition of the angular spectrum of the field [Eq. (2)] by integration over the parallel wave vector $\mathbf{K}$. When using an integration over the solid angle $\Omega$, with $k d \Omega=d^{2} \mathbf{K} / \gamma$, these factors disappear from the reciprocity relations. Nevertheless, the presence of evanescent waves would involve complex angles in the $\Omega$ representation, so that the $\mathbf{K}$ representation looks more appropriate.

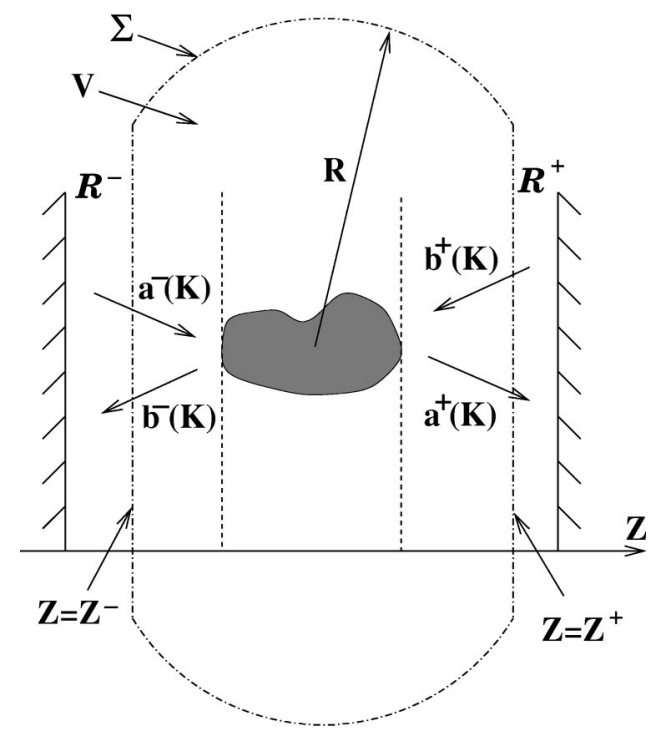

FIG. 2. Closed volume used for the application of Green's identity and the energy (or probability) balance.

\section{EXTENDED UNITARITY RELATIONS}

The second basic property of the $S$ matrix is unitarity. It is satisfied by the $S$ matrix of a lossless system (elastic scattering). It is often assumed that the unitarity condition involves only propagating waves. This belief is, in fact, based on the use of asymptotic fields in the derivation. Indeed, unitarity has been studied extensively in far-field (asymptotic) scattering [2] or scattering of source-free fields in the angularspectrum representation $[9,25]$. Conversely, the extension of unitarity relations to wave fields containing evanescent components has received little attention until now, except in the context of electromagnetic waveguide theory [24]. Nevertheless, as discussed in the Introduction of the present paper, extended unitarity relations could be helpful in various recent applications, such as time-reversed acoustics, near-field optics, or propagation through random media from the viewpoint of random-matrix theory. In this section, we show how such relations may be derived in the framework of free-space scattering from a localized potential.

In either $\mathcal{R}^{-}$or $\mathcal{R}^{+}$, the current density associated with the field is $\mathbf{J}(\mathbf{r})=A \operatorname{Im}\left\{\Psi^{*}(\mathbf{r}) \nabla \Psi(\mathbf{r})\right\}$, where $A$ is a constant, Im denotes the imaginary part, and the asterisk is the complex conjugate. With reference to Fig. 2, energy (or probability) conservation states that the total flux $\phi$ $=\int \mathbf{J} \cdot \mathbf{n} d S$ flowing outside the volume $V$ vanishes. When the radius $R$ of the sphere tends to infinity, the contribution of the flux through the portions of the sphere surface between the two planes $z=z^{-}$and $z=z^{+}$vanishes. Finally, energy (or probability) conservation reads

$$
\phi_{z^{-}}=\phi_{z^{+}}
$$

where

$$
\phi_{z^{ \pm}}=\int_{z=z^{ \pm}} \mathbf{J}\left(\mathbf{R}, z^{ \pm}\right) \cdot \mathbf{n} d^{2} \mathbf{R} .
$$


Using the angular-spectrum representation Eq. (2), the current $\phi_{z^{ \pm}}$accross a plane $z=z^{ \pm}$in $\mathcal{R}^{ \pm}$can be cast in the following form:

$$
\begin{aligned}
\phi_{z^{ \pm}}= & A \int_{K^{2} \leqslant k^{2}} \gamma\left[\left|a^{ \pm}(\mathbf{K})\right|^{2}-\left|b^{ \pm}(\mathbf{K})\right|^{2}\right] d^{2} \mathbf{K} \\
& +A \int_{K^{2}>k^{2}} \gamma\left[a^{ \pm}(\mathbf{K}) b^{ \pm *}(\mathbf{K})-a^{ \pm *}(\mathbf{K}) b^{ \pm}(\mathbf{K})\right] d^{2} \mathbf{K} .
\end{aligned}
$$

This expression for the current deserves some comment. It explicitly shows two separated contributions, one stemming from propagating waves $\left(K^{2} \leqslant k^{2}\right)$, and the other stemming from evanescent waves only $\left(K^{2}>k^{2}\right)$. Note that the latter is a crossed term between counterdecaying evanescent waves. For a given $\mathbf{K}$ such that $K^{2}>k^{2}$, if either $a(\mathbf{K})$ or $b(\mathbf{K})$ vanishes, then the associated current also vanishes. For the aim pursued in this section, it is precisely the existence of this contribution to the current that leads to the extended unitarity relations of the $S$ matrix. Introducing Eq. (11) into Eq. (9), and using the definition of the $S$ matrix (3), one obtains the three following relations, involving scattering between two propagating waves, between two evanescent waves, and between one evanescent and one propagating wave:

$$
\begin{aligned}
\int_{K^{\prime} \leqslant} & \frac{\gamma\left(\mathbf{K}^{\prime \prime}\right)}{\gamma\left(\mathbf{K}^{\prime}\right)} \mathbf{S}\left(\mathbf{K}, \mathbf{K}^{\prime}\right) \mathbf{S}^{\dagger}\left(\mathbf{K}^{\prime \prime}, \mathbf{K}^{\prime}\right) d^{2} \mathbf{K}^{\prime} \\
= & \delta\left(\mathbf{K}-\mathbf{K}^{\prime \prime}\right) \mathbf{U} \quad \text { for } K \leqslant k, K^{\prime \prime} \leqslant k, \\
= & \mathbf{S}\left(\mathbf{K}, \mathbf{K}^{\prime \prime}\right) \quad \text { for } K \leqslant k, K^{\prime \prime}>k, \\
= & \mathbf{S}\left(\mathbf{K}, \mathbf{K}^{\prime \prime}\right)-\mathbf{S}^{*}\left(-\mathbf{K},-\mathbf{K}^{\prime \prime}\right) \\
& \text { for } K>k, K^{\prime \prime}>k,
\end{aligned}
$$

where $\mathbf{U}$ is the $2 \times 2$ unit matrix. The superscript $\dagger$ denotes the conjugated and transposed matrix.

Equation (12) is the well-known unitarity condition of the $S$ matrix restricted to the homogeneous components of the fields, which was obtained previously $[2,3,25]$. Using the partitioned form of the $S$ matrix Eq. (4), this condition can be developed in terms of the generalized reflection and transmission coefficients. The resulting four expressions are the generalized Stokes relations of surface optics, which were derived in [25]. Equations (13) and (14) express, in the $S$ matrix formalism, probability (or energy) conservation in a scattering process in which the incoming and/or the outgoing fields contain evanescent components. Hence, they can be considered as extended unitarity conditions for the $S$ matrix of fields containing evanescent components. Because the $S$ matrix formalism is used in many fields of theoretical physics, and because there has been increasing interest in phenomena involving direct use and measurement of evanescent fields, we believe that this result may have important consequences and applications. For example, the development of Eqs. (13) and (14) in terms of generalized reflection and transmission coefficients leads to relationships that extend the generalized Stokes relations to evanescent fields. Such relations may be useful, e.g., in the context of surfaces, thin films, and multilayers optics. The generalized Stokes relationships and their extension to fields containing evanescent components are given in the Appendix.

\section{TIME-REVERSAL INVARIANCE}

In this section we discuss time-reversal invariance for classical scalar waves (we exclude from the discussion the question of time reversal in quantum mechanics which is difficult to separate from the measurement problem).

\section{A. Time reversal in angular-spectrum representation}

Let $\Psi(\mathbf{r}, t)$ be a classical scalar field in the time domain, and $\Psi(\mathbf{r}, \omega)$ its frequency spectrum. Because $\Psi(\mathbf{r}, t)$ is a real function, its frequency spectrum satisfies $\Psi(\mathbf{r}, \omega)$ $=\Psi^{*}(\mathbf{r},-\omega)$. From this condition, it is straightforward to show that the time-reversed field $\Psi(\mathbf{r},-t)$ has a frequency spectrum $\Psi^{*}(\mathbf{r}, \omega)$. Hence time reversal of $\Psi(\mathbf{r}, t)$ is equivalent to complex conjugation of $\Psi(\mathbf{r}, \omega)$ throughout all space. Note that time reversal is not equivalent to phase conjugation in only one plane [29]. We shall come back to this point later.

Let us see what time reversal means in terms of the angular spectrum of the monochromatic field $\Psi(\mathbf{r})$ (the variable $\omega$ is omitted in the following). From complex conjugation of Eq. (2) and the change of variable $\mathbf{K} \rightarrow-\mathbf{K}$, one obtains

$$
\begin{aligned}
\Psi^{*}(\mathbf{r})= & \int a^{*}(-\mathbf{K}) \exp \left(i \mathbf{K} \cdot \mathbf{R}-i \gamma^{*} z\right) d^{2} \mathbf{K} \\
& +\int b^{*}(-\mathbf{K}) \exp \left(i \mathbf{K} \cdot \mathbf{R}+i \gamma^{*} z\right) d^{2} \mathbf{K} .
\end{aligned}
$$

The symbols \pm have been omitted in Eq. (15) because we do not need to specify at this stage whether the field propagates in $\mathcal{R}^{+}$or in $\mathcal{R}^{-}$. We see that, in terms of angular spectrum, time reversal is equivalent to the transformation $a(\mathbf{K}) \exp (i \gamma z) \rightarrow a^{*}(-\mathbf{K}) \exp \left(-i \gamma^{*} z\right) \quad$ and $\quad b(\mathbf{K}) \exp (-i \gamma z)$ $\rightarrow b^{*}(-\mathbf{K}) \exp \left(i \gamma^{*} z\right)$ for all values of $z$ in either $\mathcal{R}^{+}$or $\mathcal{R}^{-}$. Note that Eq. (15) is valid in regions of space for which $|z|$ remains finite, so that there is no divergence of time-reversed evanescent waves when $|z|$ increases.

\section{B. Time-reversal invariance: consequence for the $S$ matrix}

In order to study the implication of time-reversal invariance for the $S$ matrix, let us consider a monochromatic field $\Psi_{1}(\mathbf{r})$ which is a solution of the scattering problem depicted in Fig. 1, the scattering potential being described by the $S$ matrix S. Let $\Psi_{2}(\mathbf{r})$ be the time reversal of the field $\Psi_{1}(\mathbf{r})$. In terms of the angular spectrum, this means that

$$
\begin{aligned}
& a_{2}^{+}(\mathbf{K}) \exp (i \gamma z)+b_{2}^{+}(\mathbf{K}) \exp (-i \gamma z) \\
& \quad=a_{1}^{+*}(-\mathbf{K}) \exp \left(-i \gamma^{*} z\right)+b_{1}^{+*}(-\mathbf{K}) \exp \left(i \gamma^{*} z\right)
\end{aligned}
$$


for all values of $z$ in $\mathcal{R}^{+}$and

$$
\begin{aligned}
& a_{2}^{-}(\mathbf{K}) \exp (i \gamma z)+b_{2}^{-}(\mathbf{K}) \exp (-i \gamma z) \\
& \quad=a_{1}^{-*}(-\mathbf{K}) \exp \left(-i \gamma^{*} z\right)+b_{1}^{-*}(-\mathbf{K}) \exp \left(i \gamma^{*} z\right)
\end{aligned}
$$

for all values of $z$ in $\mathcal{R}^{-}$. The scattering problem is invariant under time reversal if, and only if, $\Psi_{2}(\mathbf{r})$ is also a solution of the scattering problem, described by the same $S$ matrix $\mathbf{S}$. This means that the outgoing vectors $\Psi_{1}^{\text {out }}(\mathbf{K})$ $=\left[b_{1}^{-}(\mathbf{K}) \quad a_{1}^{+}(\mathbf{K})\right]$ and $\Psi_{2}^{\text {out }}(\mathbf{K})=\left[b_{2}^{-}(\mathbf{K}) \quad a_{2}^{+}(\mathbf{K})\right]$ are connected to the incoming vectors $\Psi_{1}^{\text {in }}(\mathbf{K})$

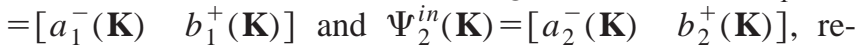
spectively, by relation (3). Introducing these conditions into Eqs. (16) and (17) leads to the following relations:

$$
\begin{aligned}
\int_{K^{\prime} \leqslant} & \mathbf{S}\left(\mathbf{K}, \mathbf{K}^{\prime}\right) \mathbf{S}^{*}\left(-\mathbf{K}^{\prime}, \mathbf{K}^{\prime \prime}\right) d^{2} \mathbf{K}^{\prime} \\
= & \delta\left(\mathbf{K}+\mathbf{K}^{\prime \prime}\right) \mathbf{U} \quad \text { for } K \leqslant k, K^{\prime \prime} \leqslant k, \\
= & -\mathbf{S}\left(\mathbf{K},-\mathbf{K}^{\prime \prime}\right) \quad \text { for } K \leqslant k, K^{\prime \prime}>k, \\
= & \mathbf{S}^{*}\left(-\mathbf{K}, \mathbf{K}^{\prime \prime}\right)-\mathbf{S}\left(\mathbf{K},-\mathbf{K}^{\prime \prime}\right) \\
& \text { for } K>k, K^{\prime \prime}>k,
\end{aligned}
$$

where the asterisk denotes the conjugated matrix. These relations express the condition of time-reversal invariance in terms of the $S$ matrix of fields containing evanescent components.

The set of Eqs. (18)-(20) is very similar to the set of Eqs. (12)-(14), which describes energy conservation. In fact, it is easy to see that these two sets of equations are equivalent, provided that the reciprocity relation (8) is satisfied. Indeed, Eqs. (18)-(20) are transformed into Eqs. (12) -(14) by using Eq. (8) and changing $\mathbf{K}^{\prime \prime} \rightarrow-\mathbf{K}^{\prime \prime}$. The result we have obtained can be summarized as follows: the condition of timereversal invariance is equivalent to both energy conservation (extended unitarity condition) and reciprocity (symmetry of the $S$ matrix). Although this result was already known for source-free fields [23], we have demonstrated that it holds for fields containing evanescent components.

\section{Time-reversal invariance and reciprocity}

The results in this paper also provide a basis to discuss the link between time-reversal symmetry and reciprocity, which is sometimes confusing in the literature [3] (see also a discussion of this point in Ref. [26]). For a scattering system in which energy is conserved [Eqs. (12)-(14) are satisfied], the conditions of time-reversal invariance [Eqs. (18)-(20)] and reciprocity [Eq. (8)] are equivalent. This is probably the reason why time-reversal symmetry and reciprocity are often mistaken. In particular, reciprocity is often presented as a consequence of time-reversal invariance [3]. This is in general incorrect. For example, we have seen that imposing time-reversal invariance leads to Eqs. (18)-(20) and not to the reciprocity condition Eq. (8). Moreover, a scattering system may be reciprocal, without being conservative [Eq. (8) is satisfied, but not Eqs. (12)-(14)]. In this case, the system is not invariant under time reversal [Eqs. (12)-(14) cannot be satisfied]. These conclusions hold for fields with or without evanescent components.

\section{Experimental feasibility of time reversal}

An important problem is the experimental achievement of time reversal in a situation involving wave scattering. In optics, the development of phase conjugating mirrors provides a practical tool to produce fields that are conjugates of each other in a given plane. Nevertheless, it has been shown that this type of phase conjugation is not equivalent to time reversal when the fields involved contain evanescent components [29]. The subject of time reversal of fields containing evanescent components is of particular interest in the context of time-reversed acoustics [22]. In this technique, the acoustic field in a direct situation is recorded on a given surface $\Sigma$, after scattering by an arbitrary object. In the reversed situation, the time-reversed field is emitted from the surface $\Sigma$, in the presence of the same scattering object. In the frequency domain, the fields in the two situations are complex conjugates of each other on $\Sigma$. Thus, this experiment is equivalent to achieving acoustic phase conjugation on the surface $\Sigma$. In both optics [21] and acoustics [22], the possibility of achieving time reversal of both the homogeneous and evanescent components of the field by phase conjugation may be questioned.

The first part of the answer is given by showing that phase conjugation on the surface of a closed cavity (or equivalently along two planes $z=z_{1}$ and $z=z_{2}$ ) is equivalent to time reversal at all points inside the cavity (or in the strip $z_{1}<z$ $<z_{2}$ ). This assertion is a consequence of the following result: two fields defined inside the strip $z_{1}<z<z_{2}$ that are complex conjugates in the two planes $z=z_{1}$ and $z=z_{2}$ are complex conjugates at all points within the strip $z_{1}<z<z_{2}$. Therefore, they are time reversed from each other in the cavity. This result holds for fields containing evanescent components. It can be derived by extending the discussion in Ref. [29] to a situation involving phase conjugation along two planes $z=z_{1}$ and $z=z_{2}$. Consequently, phase conjugation on a closed surface (or along two planes) may be a practical way to achieve complete time reversal of a field.

The second part of the answer must take into account the presence of sources inside the cavity in the direct experiment. In theory, reversing time leads automatically to the transformation of all primary sources into sinks. Therefore, to achieve time reversal experimentally, the field on the surface of the cavity has to be time reversed, and the sources have to be transformed into sinks. This is probably the greatest experimental challenge. This is also the necessary condition to obtain complete time reversal (i.e., with evanescent waves included) and achieve, for example, time-reversed focusing below the diffraction limit. The necessity of replacing sources by sinks in the time-reversed situation can be understood as follows. In the direct situation, a subwavelength source radiates a localized field whose angular spectrum con- 
tains evanescent waves. In the reversed situation, the sink is equivalent to a source with opposite phase. This localized source also radiates evanescent waves which allow the timereversed field to focus below the diffraction limit.

\section{CONCLUSION}

In summary, we have derived general properties of the $S$ matrix of fields containing evanescent components. In particular, we have shown that energy (or probability) conservation leads to relationships that extend the well-known unitarity condition of the asymptotic $S$ matrix. Using the partitioned $S$ matrix, we have shown that these relationships lead to extended Stokes relations. We have also obtained different relationships as a consequence of time-reversal invariance. On this basis, we have discussed the link between unitarity, time-reversal symmetry, and reciprocity. With the increasing interest in techniques based on measurement and control of evanescent waves, we think that this work should find broad applications. In particular, we have briefly discussed its implications in time reversal of scattered fields by phase conjugation.

\section{ACKNOWLEDGMENTS}

We wish to thank M. Fink, A. Garcia Martin, and J. L. Thomas for helpful discussions. This work was supported by the French-Spanish Integrated Program PICASSO. M.N.-V. acknowledges the support of Fundación Ramón Areces.

\section{APPENDIX: GENERALIZED STOKES RELATIONS FOR FIELDS CONTAINING EVANESCENT WAVES}

In this Appendix, we summarize the relations that are obtained by inserting the coefficients of the partitioned $S$ matrix Eq. (4) into relations (12)-(14). The first four relations are the generalized Stokes relationships obtained in Ref. [25]. The other relations are extensions of the Stokes relationships to fields containing evanescent components. We use the notations $\gamma^{\prime}=\gamma\left(\mathbf{K}^{\prime}\right)$ and $\gamma^{\prime \prime}=\gamma\left(\mathbf{K}^{\prime \prime}\right)$.

1. Relations involving homogeneous waves only

Relations valid for $K \leqslant k$ and $K^{\prime \prime} \leqslant k$ :

$$
\begin{aligned}
& \int_{K^{\prime} \leqslant k} d^{2} \mathbf{K}^{\prime}\left[\rho\left(\mathbf{K}, \mathbf{K}^{\prime}\right) \tau^{*}\left(\mathbf{K}^{\prime \prime}, \mathbf{K}^{\prime}\right)+t\left(\mathbf{K}, \mathbf{K}^{\prime}\right) r^{*}\left(\mathbf{K}^{\prime \prime}, \mathbf{K}^{\prime}\right)\right] \frac{\gamma^{\prime \prime}}{\gamma^{\prime}} \\
& \quad=0,
\end{aligned}
$$$$
\int_{K^{\prime} \leqslant k} d^{2} \mathbf{K}^{\prime}\left[\rho\left(\mathbf{K}, \mathbf{K}^{\prime}\right) \rho^{*}\left(\mathbf{K}^{\prime \prime}, \mathbf{K}^{\prime}\right)+t\left(\mathbf{K}, \mathbf{K}^{\prime}\right) t^{*}\left(\mathbf{K}^{\prime \prime}, \mathbf{K}^{\prime}\right)\right] \frac{\gamma^{\prime \prime}}{\gamma^{\prime}}
$$$$
=\delta\left(\mathbf{K}-\mathbf{K}^{\prime \prime}\right),
$$

$$
\int_{K^{\prime} \leqslant k} d^{2} \mathbf{K}^{\prime}\left[r\left(\mathbf{K}, \mathbf{K}^{\prime}\right) r^{*}\left(\mathbf{K}^{\prime \prime}, \mathbf{K}^{\prime}\right)+\tau\left(\mathbf{K}, \mathbf{K}^{\prime}\right) \tau^{*}\left(\mathbf{K}^{\prime \prime}, \mathbf{K}^{\prime}\right)\right] \frac{\gamma^{\prime \prime}}{\gamma^{\prime}}
$$$$
=\delta\left(\mathbf{K}-\mathbf{K}^{\prime \prime}\right),
$$

$$
\begin{aligned}
& \int_{K^{\prime} \leqslant k} d^{2} \mathbf{K}^{\prime}\left[r\left(\mathbf{K}, \mathbf{K}^{\prime}\right) t^{*}\left(\mathbf{K}^{\prime \prime}, \mathbf{K}^{\prime}\right)+\tau\left(\mathbf{K}, \mathbf{K}^{\prime}\right) \rho^{*}\left(\mathbf{K}^{\prime \prime}, \mathbf{K}^{\prime}\right)\right] \frac{\gamma^{\prime \prime}}{\gamma^{\prime}} \\
& \quad=0 .
\end{aligned}
$$

2. Relations involving conversion of homogeneous to evanescent waves

Relations valid for $K \leqslant k$ and $K^{\prime \prime}>k$ :

$$
\begin{aligned}
& \int_{K^{\prime} \leqslant k} d^{2} \mathbf{K}^{\prime}\left[\rho\left(\mathbf{K}, \mathbf{K}^{\prime}\right) \tau^{*}\left(\mathbf{K}^{\prime \prime}, \mathbf{K}^{\prime}\right)+t\left(\mathbf{K}, \mathbf{K}^{\prime}\right) r^{*}\left(\mathbf{K}^{\prime \prime}, \mathbf{K}^{\prime}\right)\right] \frac{\gamma^{\prime \prime}}{\gamma^{\prime}} \\
& =t\left(\mathbf{K}, \mathbf{K}^{\prime \prime}\right), \\
& \int_{K^{\prime} \leqslant k} d^{2} \mathbf{K}^{\prime}\left[\rho\left(\mathbf{K}, \mathbf{K}^{\prime}\right) \rho^{*}\left(\mathbf{K}^{\prime \prime}, \mathbf{K}^{\prime}\right)+t\left(\mathbf{K}, \mathbf{K}^{\prime}\right) t^{*}\left(\mathbf{K}^{\prime \prime}, \mathbf{K}^{\prime}\right)\right] \frac{\gamma^{\prime \prime}}{\gamma^{\prime}} \\
& =\rho\left(\mathbf{K}, \mathbf{K}^{\prime \prime}\right),
\end{aligned}
$$

$$
\begin{aligned}
& \int_{K^{\prime} \leqslant k} d^{2} \mathbf{K}^{\prime}\left[r\left(\mathbf{K}, \mathbf{K}^{\prime}\right) r^{*}\left(\mathbf{K}^{\prime \prime}, \mathbf{K}^{\prime}\right)+\tau\left(\mathbf{K}, \mathbf{K}^{\prime}\right) \tau^{*}\left(\mathbf{K}^{\prime \prime}, \mathbf{K}^{\prime}\right)\right] \frac{\gamma^{\prime \prime}}{\gamma^{\prime}} \\
& =r\left(\mathbf{K}, \mathbf{K}^{\prime \prime}\right) .
\end{aligned}
$$

$$
\begin{aligned}
& \int_{K^{\prime} \leqslant k} d^{2} \mathbf{K}^{\prime}\left[r\left(\mathbf{K}, \mathbf{K}^{\prime}\right) t^{*}\left(\mathbf{K}^{\prime \prime}, \mathbf{K}^{\prime}\right)+\tau\left(\mathbf{K}, \mathbf{K}^{\prime}\right) \rho^{*}\left(\mathbf{K}^{\prime \prime}, \mathbf{K}^{\prime}\right)\right] \frac{\gamma^{\prime \prime}}{\gamma^{\prime}} \\
& =\tau\left(\mathbf{K}, \mathbf{K}^{\prime \prime}\right) .
\end{aligned}
$$

\section{Relations involving conversion of evanescent} to evanescent waves

Relations valid for $K>k$ and $K^{\prime \prime}>k$ :

$$
\begin{aligned}
\int_{K^{\prime} \leqslant k} & d^{2} \mathbf{K}^{\prime}\left[\rho\left(\mathbf{K}, \mathbf{K}^{\prime}\right) \tau^{*}\left(\mathbf{K}^{\prime \prime}, \mathbf{K}^{\prime}\right)+t\left(\mathbf{K}, \mathbf{K}^{\prime}\right) r^{*}\left(\mathbf{K}^{\prime \prime}, \mathbf{K}^{\prime}\right)\right] \frac{\gamma^{\prime \prime}}{\gamma^{\prime}} \\
= & t\left(\mathbf{K}, \mathbf{K}^{\prime \prime}\right)-t^{*}\left(-\mathbf{K},-\mathbf{K}^{\prime \prime}\right),
\end{aligned}
$$

$$
\begin{aligned}
& \int_{K^{\prime} \leqslant k} d^{2} \mathbf{K}^{\prime}\left[\rho\left(\mathbf{K}, \mathbf{K}^{\prime}\right) \rho^{*}\left(\mathbf{K}^{\prime \prime}, \mathbf{K}^{\prime}\right)+t\left(\mathbf{K}, \mathbf{K}^{\prime}\right) t^{*}\left(\mathbf{K}^{\prime \prime}, \mathbf{K}^{\prime}\right)\right] \frac{\gamma^{\prime \prime}}{\gamma^{\prime}} \\
& =\rho\left(\mathbf{K}, \mathbf{K}^{\prime \prime}\right)-\rho^{*}\left(-\mathbf{K},-\mathbf{K}^{\prime \prime}\right),
\end{aligned}
$$

$$
\begin{aligned}
& \int_{K^{\prime} \leqslant k} d^{2} \mathbf{K}^{\prime}\left[r\left(\mathbf{K}, \mathbf{K}^{\prime}\right) r^{*}\left(\mathbf{K}^{\prime \prime}, \mathbf{K}^{\prime}\right)+\tau\left(\mathbf{K}, \mathbf{K}^{\prime}\right) \tau^{*}\left(\mathbf{K}^{\prime \prime}, \mathbf{K}^{\prime}\right)\right] \frac{\gamma^{\prime \prime}}{\gamma^{\prime}} \\
& =r\left(\mathbf{K}, \mathbf{K}^{\prime \prime}\right)-r^{*}\left(-\mathbf{K},-\mathbf{K}^{\prime \prime}\right),
\end{aligned}
$$

$$
\begin{aligned}
& \int_{K^{\prime} \leqslant k} d^{2} \mathbf{K}^{\prime}\left[r\left(\mathbf{K}, \mathbf{K}^{\prime}\right) t^{*}\left(\mathbf{K}^{\prime \prime}, \mathbf{K}^{\prime}\right)+\tau\left(\mathbf{K}, \mathbf{K}^{\prime}\right) \rho^{*}\left(\mathbf{K}^{\prime \prime}, \mathbf{K}^{\prime}\right)\right] \frac{\gamma^{\prime \prime}}{\gamma^{\prime}} \\
& =\tau\left(\mathbf{K}, \mathbf{K}^{\prime \prime}\right)-\tau^{*}\left(-\mathbf{K},-\mathbf{K}^{\prime \prime}\right) .
\end{aligned}
$$


[1] W. Heisenberg, Z. Phys. 120, 513 (1943); 120, 673 (1943).

[2] P. Roman, Advanced Quantum Theory (Addison-Wesley, Reading, MA, 1965), Chaps. 4 ( $S$ matrix formalism) and 5 (symmetry and invariance).

[3] R.G. Newton, Scattering Theory of Waves and Particles (McGraw-Hill, New York, 1966), p. 44.

[4] H.P. Stapp, Rev. Mod. Phys. 34, 390 (1962).

[5] G.F. Chew, S-Matrix Theory of Strong Interaction (Benjamin, New York, 1961).

[6] A.A. Abrikosov, L.P. Gorkov, and I.E. Dzyaloshinski, Methods of Quantum Field Theory in Statistical Physics (PrenticeHall, Englewood Cliffs, NJ, 1963), Chaps. I-III.

[7] E. Gerjuoy and D.S. Saxon, Phys. Rev. 94, 1445 (1954).

[8] D.S. Saxon, Phys. Rev. 100, 1771 (1955).

[9] M. Nieto-Vesperinas, Scattering and Diffraction in Physical Optics (Wiley, New York, 1991), Chap. 5.

[10] M.L. Mehta, Random Matrices (Academic, New York, 1991).

[11] C.W.J. Beenakker, Rev. Mod. Phys. 69, 731 (1997).

[12] E. Bascones, M.J. Calderon, D. Castelo, T. López, and J.J. Sáenz, Phys. Rev. B 55, R11 911 (1997); A. García-Martin, T. López-Ciudad, J.J. Sáenz, and M. Nieto-Vesperinas, Phys. Rev. Lett. 81, 329 (1998).

[13] R.R. Chance, A. Prock, and R. Silbey, in Advances in Chemical Physics XXXVII, edited by I. Prigogine and S.A. Rice (Wiley, New York, 1978); J.M. Wylie and J.E. Sipe, Phys. Rev. A 30, 1185 (1984); C. Henkel and V. Sandoghdar, Opt. Commun. 158, 250 (1998).

[14] C.S. West and K.A. O’Donnell, Opt. Lett. 21, 1 (1996); A.A. Maradudin, A.R. McGurn, and E.R. Méndez, J. Opt. Soc. Am. A 12, 2500 (1995).

[15] C.J. Chen, Introduction to Scanning Tunneling Microscopy (Oxford University Press, Oxford, 1993).

[16] Near-Field Optics, edited by D.W. Pohl and D. Courjon (Kluwer Academic Publishers, Dordrecht, 1993); Near-Field Nano/ Atom Optics and Technology, edited by M. Ohtsu (SpringerVerlag, Tokyo, 1998).

[17] J.M. Vigoureux, C. Girard, and D. Courjon, Opt. Lett. 14, 1039 (1989); D. van Labeke and D. Barchiesi, J. Opt. Soc. Am. A 10, 2193 (1993); C. Girard and A. Dereux, Rep. Prog.
Phys. 59, 657 (1996); J.-J. Greffet and R. Carminati, Prog. Surf. Sci. 56, 139 (1997).

[18] O. Marti, H. Bielefeldt, B. Hecht, S. Herminghaus, P. Leiderer, and J. Mlynek, Opt. Commun. 96, 225 (1993); P.M. Adam, L. Salomon, F. de Fornel, and J.P. Goudonnet, Phys. Rev. B 48, 2680 (1993); S.I. Bozhevolnyi and F.A. Pudonin, Phys. Rev. Lett. 78, 2823 (1997)

[19] D.P. Tsai, J. Kovacs, Z. Wang, M. Moskovits, V.M. Shalaev, J.S. Suh, and R. Botet, Phys. Rev. Lett. 72, 4149 (1994); S. Grésillon, L. Aigouy, A.C. Boccara, J.C. Rivoal, X. Quelin, C. Desmarest, P. Gadenne, V.A. Shubin, A.K. Saryshev, and V.M. Shalaev, ibid. 82, 4520 (1999).

[20] F. Pincemin, A.A. Maradudin, A.D. Boardman, and J.-J. Greffet, Phys. Rev. B 50, 15261 (1994); J.A. Sánchez-Gil, ibid. 53, 10317 (1996); A.V. Shchegrov, I.V. Novikov, and A.A. Maradudin, Phys. Rev. Lett. 78, 4269 (1997); S.I. Bozhevolnyi and V. Coello, Phys. Rev. B 58, 10899 (1998).

[21] S.I. Bozhevolnyi, O. Keller, and I.I. Smolyaninov, Opt. Lett. 19, 1601 (1994).

[22] M. Fink, IEEE Trans. Ultrason. Ferroelectr. Freq. Control 39 (5), 555 (1992); M. Fink, Phys. Today 50 (3), 34 (1997).

[23] J.L. Thomas, Ph.D. thesis, University of Paris VI, 1994, Chap. 4; D. Cassereau and M. Fink, IEEE Trans. Ultrason. Ferroelect. Freq. Control 39 (5), 579 (1992).

[24] C. Vassallo, Théorie des Guides d'Ondes Electromagnétiques (Eyrolles, Paris, 1985), Vol. 2, p. 169.

[25] M. Nieto-Vesperinas and E. Wolf, J. Opt. Soc. Am. A 3, 2038 (1986).

[26] R. Carminati, M. Nieto-Vesperinas, and J.-J. Greffet, J. Opt. Soc. Am. A 15, 706 (1998).

[27] L. Kazandjian, J. Acoust. Soc. Am. 92, 1679 (1992).

[28] H.A. Lorentz, Versl. Gewone Vergad. Afd. Natuurkd. K. Ned. Akad. Wet. 4, 176 (1896); Lorentz, Collected Papers (Nijhoff, Den Haag, 1936), Vol. III. See also M. Nieto-Vesperinas, Scattering and Diffraction in Physical Optics (Ref. [9]), Chap. 1, p. 8.

[29] M. Nieto-Vesperinas and E. Wolf, J. Opt. Soc. Am. A 2, 1429 (1985). See also M. Nieto-Vesperinas, Scattering and Diffraction in Physical Optics (Ref. [9]), Chap. 8. 